\title{
Notes méthodologiques à partir d'une étude de cas fennique
}

Methodological notes inspired by a Baltic Finnic case study

Metodoloogilisi märkmeid ühe läänemeresoome keele uurimuse varal

Jean Léo Léonard

\section{(c) OpenEdition}

Journals

Édition électronique

URL : https://journals.openedition.org/efo/605

DOI : 10.4000/efo.605

ISSN : 2275-1947

Éditeur

INALCO

Édition imprimée

Date de publication : 1 décembre 2012

ISBN : 978-2-343-02592-6

ISSN : 0071-2051

Référence électronique

Jean Léo Léonard, « Notes méthodologiques à partir d'une étude de cas fennique », Études finno-

ougriennes [En ligne], 44 | 2012, mis en ligne le 14 février 2014, consulté le 10 juillet 2021. URL : http:// journals.openedition.org/efo/605; DOI : https://doi.org/10.4000/efo.605

Ce document a été généré automatiquement le 10 juillet 2021.

\section{(c) (7) (8)}

Études finno-ougriennes est mis à disposition selon les termes de la Licence Creative Commons Attribution - Pas d'Utilisation Commerciale 4.0 International. 


\title{
Notes méthodologiques à partir d'une étude de cas fennique
}

\author{
Methodological notes inspired by a Baltic Finnic case study \\ Metodoloogilisi märkmeid ühe läänemeresoome keele uurimuse varal
}

Jean Léo Léonard

\section{Introduction}

1 L'objectif de cet article est de revisiter nombre de concepts-clés de la sociolinguistique à la lumière de considérations relatives aux langues fenniques méridionales proches de l'estonien, qui ont émergé depuis deux décennies : võro, seto, mulgi, kihnu. Le seul fait que ces variétés de fennique méridional, longtemps considérées comme des dialectes, soient aujourd'hui promues au rang de langues (les sociolinguistes picards utiliseraient, en pensant à des langues comme le picard, dans une situation analogue, le terme de langues collatérales), est en soi un phénomène qui pousse à revisiter la table des valeurs sociolinguistiques attribuables à des composantes du répertoire. Nous allons donc entreprendre à partir de cet exemple, qui nous fournira une étude de cas, un itinéraire critique parmi les notions fondamentales associées au contact de langues et à la sociologie du langage. L'idée de rédiger une contribution allant dans ce sens est également née de la lecture critique de nombre d'articles publiés dans le présent volume. Il nous a semblé opportun de remettre en cause la lecture des concepts et des données, aussi bien quantitatives que qualitatives, dans un numéro spécial de la revue E.F.O. consacré à la sociolinguistique des langues ouraliennes, notamment parce que nous sommes convaincu que le domaine ouralien est riche en faits déconcertants pour les conceptions ordinaires de la sociolinguistique, du moins celle pratiquée de ce côté-ci de l'Atlantique, le plus souvent nourrie de lectures nord-américaines ou centrée sur les "grands» pays d'Europe occidentale, dont les langues sont véhiculaires (anglais, allemand, français, espagnol, mais également italien, dans une certaine mesure, dans le registre culturel) et dont les procédés formatifs, en termes de construction nationale, diffèrent sensiblement de ceux que l'on peut observer dans des pays ou des territoires 
où des langues finno-ougriennes se sont individuées en langues nationales au cours de l'histoire ${ }^{1}$.

Dialectes et langues d'Estonie

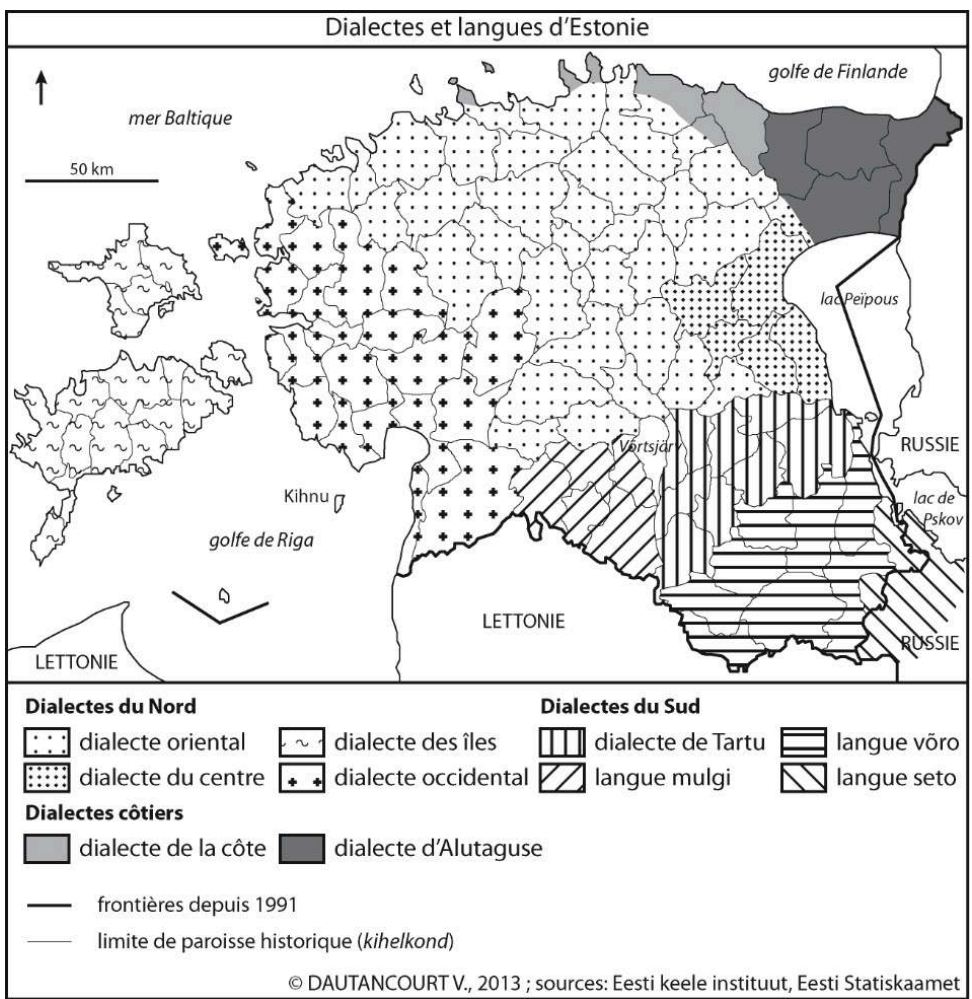

2 L'argumentation procédera en deux temps : dans la section 2, le relativisme des formes et des contenus du champ de contacts ou de conflits de variétés et de langues présentes dans un même territoire au cours de l'histoire sera développé, à des fins de modélisation qualitative - étape indispensable à toute analyse quantitative de données relatives au contact ou au conflit de langues. Dans la section 3, nous tenterons de suggérer des pistes pour lire et interpréter les données quantitatives, en comparant les données déclaratives en Galice (Espagne) et en Võru (Estonie) sur les pratiques langagières selon les domaines d'usage. Nous verrons que la trame des relations entre groupes sociaux, définissables selon le revenu ou le niveau d'éducation, mais aussi en fonction de la nature plus ou moins diversifiée de leurs répertoires (bilingues actifs, passifs) et de leurs stratégies adaptatives en tant qu'agents ou « joueurs » (alternances codiques et choix de registres sur le continuum diaphasique, etc.) permet de donner plusieurs lectures à un ensemble de données, ou en croisant diverses variables. Enfin, nous tenterons de montrer que les apories de l'analyse en termes de catégories a priori, telles que fonction vernaculaire, véhiculaire, référentiaire, etc., trouvent une résolution dans la lecture herméneutique - c'est-à-dire interprétative, contextualisante - des données, s'attachant à une lecture non seulement des données elles-mêmes, mais aussi des comportements observables autour, en dehors et au-delà des données chiffrées. Rien ne peut se décider dans l'abstrait, bien que nous partions de concepts abstraits en première analyse. En confrontant ces abstractions à des faits réels (même s'ils relèvent du domaine subjectif de la déclaration), les catégories finissent, en deuxième analyse, par s'auto-organiser en modèles interprétatifs plus fiables. 


\section{Relativisme des formes et des statuts}

3 Nous reprendrons à Tiit Hennoste sa chronologie de l'histoire sociale de la langue estonienne (Hennoste 1997) ${ }^{2}$. Cette périodisation nous servira de boussole et de cadre général pour mesurer les enjeux de l'individuation tardive des variétés dialectales estoniennes méridionales à la jointure entre le $\mathrm{xx}^{\mathrm{e}}$ et le $\mathrm{xxI}$ siècle. En effet, cette émergence tardive est l'un des phénomènes qui suggèrent que la sociolinguistique - et notamment sa dimension glottopolitique - réserve au chercheur des imprévus. Que des variétés vernaculaires considérées comme des sociolectes au plus bas de l'échelle, y compris dans le contexte de "l'éveil national » estonien, acquièrent droit de cité, reçoivent une codification et une standardisation (autrement dit une normativisation) soutenues et financées par l'État, à travers l'activité appliquée des linguistes et dialectologues de l'ère postcommuniste, cela n'a rien d'anodin. Ces variétés, comme nous allons le voir, n'avaient jamais auparavant été considérées comme «nobles » ou représentatives de l'image que les élites pouvaient se faire de la construction nationale estonienne. Pas même comme matériaux constitutifs, puisque les réformateurs, comme Johannes Aavik, ne les tenaient aucunement en considération - bien au contraire (cf. Chalvin 2010). Tout au plus avaient-elles une fonction stylistique en littérature, ce qui ne retire rien à leur statut subalterne sur le plan social, et sur leur relative illégitimité à contribuer à la construction nationale sur le plan référentiaire.

4 Nous utiliserons les termes suivants afin de caractériser les variétés ou langues des répertoires en usage au cours de l'histoire, selon un modèle implicitement fonctionnaliste (chaque valeur qualifiante pouvant également être appelée "fonction »: vernaculaire, référentiaire, véhiculaire, confessionnelle et corporatiste. Chacun de ces termes n'est jamais qu'une étiquette très approximative - et c'est pourtant de ce genre de termes portemanteaux dont se sert couramment la sociolinguistique. Dans l'ordre, vernaculaire désigne la fonction la plus ordinaire ou de plus grande proximité, située comme «basse » dans la hiérarchie sociale, à savoir la langue du cercle familial, plus précisément la langue des pauvres ou des classes les plus modestes. Une telle définition, sans euphémisme, laisse déjà entrevoir toutes ses faiblesses - on peinerait à $\mathrm{y}$ trouver un concept transparent, au-delà du simple jugement social. La fonction référentiaire est celle du cadre institutionnel, par exemple l'État-nation constitué en tant que terme de référence majeure, ou une identité émergente ou déjà constituée en tant que terme de référence mineure, au titre d'une identité locale ou de groupe. La fonction référentiaire est dotée d'une certaine légitimité, autrement dit d'une forme de prestige, plus ou moins formelle ou déclarée selon les contextes géopolitiques et historiques. La fonction véhiculaire, qui n'est guère plus simple à définir, suppose que la langue détient une fonction de communication élargie, entre États-nations ou entre entités constitutives d'un État-nation hégémonique - mais on sait combien la formation d'une langue comme le russe, aujourd'hui véhiculaire dans sa sphère d'influence, ne s'est véritablement réalisée qu'à date très récente, après être longtemps resté une variété concurrente du slavon d'église. La fonction confessionnelle, précisément, est celle de la langue d'église, qui implique le plus souvent des propriétés structurales caractéristiques, sur le plan de la forme aussi bien grammaticale que lexicale et stylistique (emprunts, calques, phraséologie spécifique, variabilité en fonction des scripta et des scripteurs, etc.). La fonction corporatiste permet tant bien que mal de définir les contours sociaux d'une variété dialectale ou d'une langue parlée par un 
groupe social (classe, caste) défini sur le plan des intérêts socioéconomiques, ou selon sa position sur le spectre social - on pourrait aussi bien parler de fonction congrégative, mais le risque de confusion avec le congrégationisme religieux est trop grand. Aucun de ces termes n'est satisfaisant, mais chacun est cependant bien plus approprié qu'une quelconque valeur définie subjectivement par le chercheur sur l'échelle «Haut » versus «Bas », sur laquelle se fonde la théorie de la diglossie (Ferguson 1959)3.

Enfin, le dernier terme qualifiant sera «collatéral ». En bref, on désigne par ce terme des langues proches, longtemps en situation de statut dialectal, qui s'avèrent, par le jeu de l'histoire sociale de la légitimation des registres et variétés d'un diasystème ou des répertoires, avoir été reléguées sur l'échiquier glottopolitique de la construction nationale à un statut non pas central, mais collatéral, en termes de fonction et d'usage. Nous avons personnellement insisté sur la teneur glottopolitique potentielle de ce terme (Léonard 2004). On ne peut utiliser ce terme que pour des langues proches - les langues collatérales sont donc des variétés basses d'une diglossie de type fergusonien ayant bénéficié d'une revalorisation, ou d'une réhabilitation de leur statut accompagnée le plus souvent d'une élaboration substantielle de leur corpus (lexique et grammaire).

6 Il semble bien que l'État-nation estonien, dont l'indépendance a été restaurée en 1991, comprenne l'utilité stratégique de développer des initiatives en faveur de la diversification des formes de la langue nationale dans ses régions périphériques et rurales. Mais ce n'est pas le seul facteur en jeu : dans ce cas de figure, la société civile a joué le jeu, recomposant des réseaux et des communautés de pratique liées à l'animation socioculturelle et au maintien d'une sociabilité locale, dans des zones rurales en crise à la suite de la décollectivisation de l'agriculture et de la privatisation des entreprises - une société civile qui, au sortir du communisme, ne se laissait pas facilement convaincre de la nécessité d'initiatives collectives et participatives, comme le requiert la revitalisation en situation d'élaboration d'une langue de statut vernaculaire. Les journaux en langues collatérales estoniennes méridionales (Uma Leht à Võru, Peko Helü en région Setu, Kyne à Kihnu, Üitsainus Mulgimaa dans l'aire Mulgi, etc.) couvrent des manifestations festives, des réunions, des colloques et assemblées, des cérémonies folkloriques ou commémoratives, qui sont autant de cadres participatifs de l'activité sociale.

7 Afin d'illustrer combien les catégories sociolinguistiques (en termes d'éléments constitutifs des répertoires langagiers), mais aussi glottopolitiques (en termes de langues en contact ou en concurrence sur le "marché linguistique») ne sont pas définies ni définissables de manière immanente, ni même de manière aussi tranchée et univoque que le chercheur pourrait le souhaiter, nous allons mettre le modèle fonctionnaliste à l'épreuve des faits historiques. Pour ce faire, nous allons utiliser, sous une version remaniée et réinterprétée, la chronologie de l'évolution sociolinguistique de l'estonien proposée il y a désormais plus d'une quinzaine d'années par Tiit Hennoste (1997) - les « sociopériodes ", ou phases socioévolutives de l'estonien en relation avec sa construction nationale, qui sont au nombre de neuf, selon ce modèle.

Périodisation par Tiit Hennoste (1997)

\begin{tabular}{|l|l|l|l|}
\hline Phase & Période & Caractérisation & Acronyme \\
\hline
\end{tabular}




\begin{tabular}{|c|c|c|c|}
\hline I & Jusqu'au XIII ${ }^{\mathrm{e}}$ siècle & Tripartition dialectale & [TriDial] \\
\hline II & $\begin{array}{l}\text { Du début du XIII siècle aux XVI } \\
\text { et XVII }{ }^{\mathrm{e}} \text { siècles }\end{array}$ & $\begin{array}{l}\text { Hégémonie bas-allemande et } \\
\text { instauration du féodalisme }\end{array}$ & [Hég.BA] \\
\hline III & $\begin{array}{l}\text { Des } \mathrm{XVI}^{\mathrm{e}}-\mathrm{XVII}{ }^{\mathrm{e}} \text { siècles au début } \mathrm{du} \\
\mathrm{XVIII}^{\mathrm{e}} \text { siècle }\end{array}$ & $\begin{array}{l}\text { Luthérianisme, hégémonie suédoise et } \\
\text { essor du haut-allemand }\end{array}$ & [Hég.Suéd.] \\
\hline IV & $\begin{array}{l}\text { Du début du XVIII siècle aux } \\
\text { années } 1860\end{array}$ & $\begin{array}{l}\text { Hégémonie russe, émergence d'une } \\
\text { culture écrite de l'estonien }\end{array}$ & [Hég.Rus.] \\
\hline $\mathrm{V}$ & Années 1860-1870 & $\begin{array}{l}\text { "Éveil national», essor de l'estonien } \\
\text { septentrional }\end{array}$ & [Év.Nat.] \\
\hline VI & $\begin{array}{l}\text { Des années } 1880 \quad \text { aux } \\
\text { années } 1914-1920\end{array}$ & Russification, configuration tripolaire & [Conf.Tripol.] \\
\hline VII & $\begin{array}{l}\text { Des années } 1914-1920 \quad \text { à } \\
1940-1944\end{array}$ & Estonie indépendante & [Est.Indép.] \\
\hline VIII & De 1940-1944 aux années 1980 & $\begin{array}{l}\text { Annexion soviétique et russification } \\
\text { démographique }\end{array}$ & [Hég.Sov.] \\
\hline IX & Depuis les années 1980 & Estonie européenne globalisée & [Est.Eur.Glob.] \\
\hline
\end{tabular}

Derrière chaque caractérisation de période, on pensera aux effets de ces changements sociopolitiques et culturels sur le statut et la fonction des différents dialectes composant le réseau dialectal estonien. On pensera aussi à la relation qu'entretient chaque terme du répertoire avec les autres variétés ou langues constitutives.

La périodisation proposée par Tiit Hennoste se résume aux étapes suivantes ${ }^{4}$ :

10 Phase I [TriDial] : de l'âge du fer à la fin du XIII siècle. La population estonienne, culturellement divisée entre trois régions, ne semble pas assujettie à une hégémonie étrangère, mais les données de l'archéologie montrent l'intersection et l'imbrication de nombreuses cultures, entre populations baltes, scandinaves, fenniques, puis slaves. La bipartition nord-sud du réseau dialectal doit dater d'avant l'ère chrétienne, tandis que la tripartition entre les aires nord-orientale, septentrionale et méridionale a dû se consolider à la fin de cette phase. L'aire où un complexe politique et social semble le plus organisé, notamment sur le plan de la défense et des échanges commerciaux, est l'archipel de Saaremaa, sur le littoral occidental ${ }^{5}$.

11 Phase II [Hég.BA] : suite à la croisade menée initialement par les Danois au XIII siècle commence l'hégémonie bas-allemande en Estonie et dans la partie septentrionale des Pays baltes, qui instaure une division socioéconomique et politique entre la population de langue bas-allemande, dont la langue constitue le terme « haut » du répertoire, et la population de langue estonienne, en bas de l'échelle sociale. L'estonien revêt la fonction d'un sociolecte, ou variété basse dans l'échelle diglossique - la population autochtone estonophone fournit la main-d'œuvre du féodalisme balte, dominé par les seigneurs et les moines-soldats germanophones. Le bas-allemand entre en contact par superposition avec les dialectes estoniens, influençant profondément leur grammaire 
et leur lexique (nombreux emprunts et calques). Trois facteurs mettent fin à cette période: a) l'essor du luthérianisme comme modèle confessionnel dominant, b) l'hégémonie suédoise et c) la prépondérance socioculturelle du haut-allemand, qui prend cette fois le pas sur le bas-allemand, qui dominait politiquement et socialement jusqu'alors.

Phase III [Hég.Suéd.] : des textes sont écrits en estonien, tandis que la part du latin diminue dans les usages écrits, au profit de l'allemand, mais aussi, désormais, du suédois. Deux variétés d'allemand sont de plus en plus en usage : le haut-allemand, en tant que langue religieuse et administrative, et le bas-allemand, en registre moyen, voire bas - autrement dit, le premier dialecte (le haut-allemand) revêt une fonction référentiaire et confessionnelle, tandis que le deuxième (le bas-allemand) est utilisé dans la vie quotidienne, à titre corporatiste (le cercle des négociants et des propriétaires terriens) ou vernaculaire. La langue la plus prestigieuse, dans sa gamme de variétés, demeure l'allemand, suivi par le suédois, tandis que l'estonien reste au bas de l'échelle. Un registre confessionnel d'estonien littéraire prend forme, fortement imprégné d'allemand: la majeure partie des textes produits sont des traductions, donc éminemment sujettes aux calques et aux emprunts. À la fin de la troisième période, $10 \%$ des Estoniens sont alphabétisés, grâce au séminaire Forselius, qui a formé les maitres des écoles rurales à travers lesquelles se diffusent les textes traduits en estonien. L'écart se creuse, durant cette période, entre la langue écrite et la langue de la " tradition orale", notamment des chants populaires : le registre littéraire se divise en registre « haut ", imprégné d'allemand, et registre «bas », relativement autonome. Les deux variétés principales d'estonien, nord et sud, sont littéralisées par les pasteurs luthériens, durant cette période, en fonction des besoins de communication locaux.

Phase IV [Hég.Rus.] : cette période commence par l'annexion de l'Estonie à la Russie et la publication de la bible en estonien septentrional, en 1739 (principalement traduite par A. Thor Helle, 1683-1748). L'allemand reste indispensable, le suédois disparaît de l'usage cultivé, tandis que le russe prend sa place dans l'élite régionale. Le latin est devenu obsolète dans la vie administrative, si bien que l'allemand et l'estonien se retrouvent aux deux extrêmes de la polarité formel/informel, avec le haut-allemand comme variété la plus prestigieuse. Il faut distinguer deux variétés d'estonien : celui en usage chez les Estoniens et celui pratiqué par les germanophones. La culture écrite se répand chez les Estoniens autochtones, au gré des traductions de calendriers, de textes d'éducation morale et technique, etc. L'estonien commence à prendre une autre teneur psychosociale que dans les siècles passés, où il était cantonné à un statut purement vernaculaire.

Phase V [Év.Nat.] : cette période est celle de "l'éveil national », qui prend forme alors que l'allemand est plus fort que jamais en tant que langue de l'espace public, qu'il s'agisse du haut-allemand, langue de prestige et de l'administration, ou du basallemand des barons baltes, qui tend à devenir un vernaculaire régional, en position diastratique $^{6}$ intermédiaire. Bien que la science et l'éducation supérieure se développent en allemand, on publie de plus en plus en estonien, notamment à travers la presse. Les formes de chant traditionnel, aussi bien que l'estonien d'église, entrent en déclin profond. De plus en plus, une polarité diglossique émerge, distinguant les variétés dialectales et la variété littéraire d'estonien, réparties entre variété haute (référentiaire) et variété basse (vernaculaire). Cette polarité diglossique, de type fergusonien, se développe parallèlement à la polarité diglossique de type fishmanien, 
qui oppose l'allemand à l'estonien - en particulier l'estonien dialectal. Le bilinguisme allemand-estonien se généralise comme jamais auparavant. Le grammairien estonien Ferdinand Johann Wiedemann (1805-1887) publie en 1864 une description grammaticale du võro (rééditée en 2002$)^{7}$ et, en 1875 , une colossale grammaire de l'estonien (rééditée en 2011).

Phase VI [Conf.Tripol.] : la russification prend le pas sur les formes de diglossie ou de dilalie $^{8}$ entre variétés germaniques et fenniques qui ont peu à peu émergé au cours des phases précédentes. La revendication nationaliste estonienne prend un caractère plus radical. Le russe devient langue officielle dans tous les domaines de la vie publique (éducation, sciences, administration). À l'estonien littéraire sont concédés les domaines de l'enseignement primaire, de la littérature nationale, du journalisme et de l'éducation populaire. La dilalie émergente a fait place à une tripartition référentiaire, tandis que persistent les niveaux diglossiques hérités des périodes précédentes (l'estonien dialectal se trouvant évidemment au bas de l'échelle). Durant cette période, le corpus de l'estonien littéraire s'épure des éléments germaniques qui imprégnaient l'estonien confessionnel, tandis que des éléments dialectaux ou conçus par des réformateurs de la langue, comme Aavik ou Veski, viennent alimenter ce remaniement, en fonction de koinès locales (cependant, Aavik n'est guère favorable aux dialectes, sauf à des fins stylistiques).

La phase VII [Est.Indép.] est celle de l'indépendance de l'Estonie, de 1920 à 1940-1944, durant laquelle l'estonien littéraire devient langue nationale. La langue fait l'objet d'un intense travail d'élaboration dans tous les domaines de son corpus: terminologie, orthographe, morphologie, expression écrite. L'idée qu'il n'existe qu'une seule et unique variété d'estonien - la langue littéraire, basée sur la variété septentrionale et sur le travail des réformateurs de la langue - s'impose durablement. Ce registre ne se limite plus à l'écrit, mais devient également la norme pour l'expression orale dans le domaine public. La relation diglossique se durcit entre la variété littéraire, septentrionale, et les variétés dialectales régionales, cantonnant les dialectes à l'usage familial : leur vernacularisation passe par une ségrégation psychosociale, qui leur nie tout droit de cité dans l'espace public formel. L'estonien méridional disparaît de l'usage littéraire, sauf dans le registre poétique ou dans les textes religieux de tradition locale. La connaissance du russe s'amenuise dans une société où il n'était que peu implanté, de même que les autres formes de bilinguisme - les lycées deviennent les principaux vecteurs, davantage formels, du bilinguisme.

Phase VIII [Hég.Sov.] : durant l'annexion soviétique, de 1940-1944 à 1991, l'Estonie perd une partie de ses habitants suite à l'exode de populations issues tant des villes que des campagnes et fuyant le nouveau régime, et aux déportations en Sibérie. À la fin de la période VIII, un tiers de la population environ est russophone, et n'utilise pratiquement que le russe dans la vie quotidienne et dans l'espace public - bien qu'une proportion de russophones ait une attitude favorable à l'estonien et à sa culture. Bien qu'ayant préservé un statut officiel aux côtés du russe, l'estonien n'est pas avantagé, et la population estonophone, qui peine à intégrer le russe dans son répertoire, se sent en situation de ségrégation face aux russophones nouvellement installés. De nombreux registres de langue disparaissent ou s'affaiblissent durablement. L'estonien administratif rigidifie sa forme stylistique au contact du russe de l'administration soviétique - il est désormais pénétré de calques russes, comme il fut imprégné de calques germaniques par le passé. Les dialectes ruraux, affaiblis par la collectivisation 
et par les déportations ou l'exode de la fin des années 1940, s'éteignent progressivement ou doivent résister à un renforcement de la ségrégation psychosociale, conformément à l'idéologie soviétique, qui considère les dialectes avec mépris. Du point de vue des configurations diglossiques fergusoniennes, deux ensembles émergent, rétrospectivement : a) les îles des archipels et la région de Võru, où le dialecte local est encore couramment utilisé au quotidien; b) les autres régions d'Estonie, où les dialectes ont fusionné avec l'estonien littéraire.

Phase IX [Est.Eur.Glob.] : on assiste à une re-estonisation volontariste de l'État, dans le cadre de la restauration de l'indépendance en 1991. Trois changements marquants modifient le paysage psychosocial des phases précédentes $^{9}: 1$ ) les variétés orales prennent le devant de la scène, au détriment de la langue littéraire ; 2 ) le prestige et l'utilité de l'estonien décroissent face à la concurrence des langues internationales, notamment face à l'anglais; 3) l'estonien s'imprègne de calques issus des langues véhiculaires européennes, probablement plus que jamais auparavant. La langue littéraire ne prime plus de manière aussi indiscutable sur les dialectes, qui refont surface dans le paysage linguistique de l'Estonie postsoviétique. La langue d'État, ou langue nationale n'a plus le même prestige dans un monde globalisé, au sein d'un espace européen sans frontières.

19 Maintenant que cette chaîne séquentielle a été présentée dans ses grandes lignes, nous allons pouvoir lui appliquer de manière critique les fonctions du répertoire, tout au long du processus de construction nationale de l'Estonie. Nous allons en quelque sorte tester sur chaque étape de cette périodisation la validité ou le caractère heuristique aussi bien de l'échelle de valeurs diglossiques (variétés hautes versus basses) que les fonctions (vernaculaire, véhiculaire, référentiaire, corporatiste, confessionnelle). L'exercice sera d'autant plus salutaire sur le plan méthodologique que de nombreuses zones d'indécision émergeront au cours de cette tentative de catégorisation dans une perspective de sociolinguistique historique.

La phase I est, a posteriori, une zone grise pour l'analyse sociolinguistique : on ne pourra rien en dire, faute de documents tangibles. C'est le domaine légitime de l'archéologue et de l'historien. Nous ne pourrions que spéculer de manière ingénue - pour ne pas dire caricaturale - si nous prenions le risque de catégoriser les variétés et les contacts de langues durant cette période ${ }^{10}$.

21 La phase II, [Hég.BA], permet de poser une superposition diglossique élémentaire relevant de la diglossie de Fishman -, si l'on exclut du tableau la langue dite de culture encore en usage dans la composante ecclésiastique européenne de l'époque, à savoir le latin :

Tableau 1.1. Diglossie de la phase II de Hennoste [Hég.BA]

\begin{tabular}{|l|l|}
\hline Haute & Bas-allemand \\
\hline Basse & Réseau dialectal estonien \\
\hline
\end{tabular}

Cependant, dans le sud de l'Estonie, il faut aussi compter avec la présence du latin ainsi que du polonais. Peut-on leur attribuer la valeur de variétés hautes pour autant? 
Si l'on suit à la lettre l'esprit de la hiérarchie diglossique, la phase III [Hég.Suéd.] se laisserait-elle décrire comme dans le tableau 1.2 ?

Tableau 1.2. Diglossie de la phase III de Hennoste [Hég. Suéd.]

\begin{tabular}{|l|l|l|}
\hline Haute? & Haut-allemand & Confessionnel \\
\hline & Suédois, latin & Référentiaire \\
\hline & Bas-allemand & Corporatiste \\
\hline & Koinè de Tallinn & Confessionnel \\
\hline Basse & Koinè de Tartu & Confessionnel \\
\hline
\end{tabular}

Quelles sont véritablement la place et la fonction du suédois dans cette province périphérique qu'est l'Estonie, sachant qu'en Suède même le latin tient encore lieu de langue de culture, voire de langue de chancellerie? Devrait-on le classer comme "Moyen-Haut " afin de rendre compte à chaque étage de la hiérarchie d'un niveau discret? Nous nous abstiendrons de noter quoi que ce soit dans la colonne de gauche. L'essor du haut-allemand en tant que langue de la doctrine luthérienne relève surtout du livre et de l'usage écrit confessionnel. Cela suffit-il à conférer la valeur "variété haute ", dans une périphérie comme l'Estonie, à cette langue d'église en cours d'expansion? Certes, dans les pays de langue allemande, le haut-allemand s'imposera peu à peu également comme langue de chancellerie. Mais le bas-allemand, langue que l'on suppose davantage cantonnée à l'usage oral qu'écrit dans la classe des propriétaires terriens et des gens des bourgs au service de cette élite agraire, est-il pour autant « rétrogradé » face au haut-allemand ? Peut-on maintenir la teneur discrète des variétés sur l'échelle diglossique ? Une solution - qui n'est cependant qu'un pis-aller consiste à attribuer des valeurs ou des qualités ${ }^{11}$, en partie ad hoc, mais explicites sur le plan de l'analyse, à ces variétés constitutives du répertoire : registre confessionnel pour le haut-allemand, valeur référentiaire pour le suédois et le latin - ce dernier étant d'ailleurs à la fois véhiculaire dans son champ d'usage lettré et diplomatique, et référentiaire en tant que langue du catholicisme, qualité que renforce encore le contraste avec le haut-allemand, langue du protestantisme. Mais comment qualifier le bas-allemand? En tant que langue de l'élite agraire, on serait tenté d'y voir un registre en usage au sein d'une corporation, et d'utiliser le terme de registre corporatiste, mais n'est-ce pas là encore une qualification réductrice, voire abusive?

Lors de la phase IV [Hég.Rus.], on peut s'attendre à une échelle encore plus difficile à définir verticalement : comment y classer le russe, qui n'a qu'une fonction marginale, en dépit de l'hégémonie russe qui ne fait que commencer, sans entrer encore dans une politique de russification? Dans quelle mesure le suédois reste-t-il référentiaire? La koinè septentrionale de l'estonien, cependant, commence quant à elle à revêtir une fonction référentiaire - elle commence à être un argument de la construction nationale estonienne. Les autres dialectes restent résolument vernaculaires, et la koinè méridionale de Tartu est déjà obsolète depuis la fin de la période III. 
Tableau 1.3. Diglossie de la phase IV de Hennoste [Hég.Rus.].

\begin{tabular}{|l|l|l|}
\hline Haute & Haut-allemand & Confessionnel, Véhiculaire \\
\hline & Russe, Suédois & Référentiaire \\
\hline & Bas-allemand & Corporatiste \\
\hline & Koinè de Tallinn & Confessionnel et référentiaire \\
\hline Basse & Réseau dialectal estonien & Vernaculaire \\
\hline
\end{tabular}

La période $\mathrm{V}$ est quasiment identique à la précédente, si ce n'est que la koinè septentrionale voit son caractère référentiaire, en tant qu'emblème, voire vecteur de la nation, considérablement renforcé. La période VI voit un jeu de chaises musicales se mettre en place: le russe remonte l'échelle diglossique pour devenir la principale variété référentiaire... de l'hégémonie étrangère, face aux langues de plus ancienne implantation qui le concurrencent sur un territoire qui est en voie de s'émanciper en tant que petite nation européenne, dans le cadre de l'éveil des nationalismes de l'époque dite «libérale ». Il y a désormais trois langues référentiaires en concurrence : le russe, intrusif, le haut-allemand, relevant de la superstructure (culture, diplomatie, religion), et l'estonien septentrional, qui devient un protagoniste à part entière de la construction nationale.

Tableau 1.4. Diglossie de la phase V de Hennoste [Év.Nat.].

\begin{tabular}{|l|l|l|}
\hline Haute & Russe & Référentiaire \\
\hline & Haut-allemand & Référentiaire et véhiculaire \\
\hline & Bas-allemand & Corporatiste \\
\hline & Koinè de Tallinn & Référentiaire \\
\hline Basse & Réseau dialectal estonien & Vernaculaire \\
\hline
\end{tabular}

Dans la phase VII [Est.Indép.], la hiérarchie se simplifie, et la gamme des variétés du répertoire national renforce ses contrastes : l'estonien est désormais le seul référentiaire national, ainsi que la langue d'usage de la majorité de la population. Le haut-allemand est disponible comme langue véhiculaire (langue académique et des échanges économiques régionaux vers l'ouest), aux côtés d'autres langues véhiculaires européennes - qu'il n'y aurait guère de sens à inclure dans le tableau, en raison de leur externalité. Les communautés suédophone de Saaremaa, russe du lac Peipus ou parlant la variété bas-allemande héritée des phases précédentes continuent de faire usage de ces langues, mais au même titre que les Estoniens des périphéries continuent de parler leurs vernaculaires. 
Tableau 1.5. Diglossie de la phase VII de Hennoste [Est.Indép.].

\begin{tabular}{|l|l|l|}
\hline Haute & Estonien & Référentiaire \\
\hline & Haut-allemand & Véhiculaire \\
\hline Basse & Réseau dialectal estonien, Bas-allemand, suédois, russe, etc. & Vernaculaire \\
\hline
\end{tabular}

La phase VIII [Hég.Sov.] change de nouveau la donne, en rétablissant le russe comme variété haute, sans priver pour autant l'estonien de sa qualité de langue référentiaire, co-officielle, mais en position plus basse que le russe, langue véhiculaire à la fois en tant que langue de la deuxième puissance mondiale et à l'échelle de la territorialité multinationale de l'Union soviétique. Il est intéressant de constater que la position de l'estonien face au russe n'est pas comparable à celle que l'on pouvait observer dans les phases IV [Hég.Rus.] ou V [Év.Nat.], bien qu'elle rappelle la situation de la phase VI [Conf.Tripol.], mais qu'il ne s'agit pas non plus d'une configuration tripartite de trois langues référentiaires, puisque toutes les populations germanophones (ou suédophones, par ailleurs) ont quitté le pays lors de l'annexion de l'Estonie par l'Union soviétique. D'ailleurs, ce n'est ni le même estonien que dans les périodes précédentes - en tant que langue fonctionnelle et constituée dans son corpus et ses fonctions de langue nationale (avec un nationalisme désormais réprimé, supposé se transcender à travers l'internationalisme prolétarien, aux conditions géostratégiques de la superpuissance soviétique qui désormais exerce une hégémonie totale) - ni le même russe qui se retrouvent face à face : le nageur ne se baigne pas deux fois dans la même rivière.

Tableau 1.6. Diglossie de la phase VIII de Hennoste [Hég.Sov.].

\begin{tabular}{|l|l|l|}
\hline Haute & Russe & Véhiculaire et référentiaire \\
\hline & Estonien & Référentiaire \\
\hline Basse & Réseau dialectal estonien & Vernaculaire \\
\hline
\end{tabular}

Enfin, la phase IX [Est.Eur.Glob.] est celle de la restauration de l'Estonie indépendante. L'estonien reprend sa place de principale langue référentiaire, tandis que le russe prend une valeur principalement véhiculaire, aux côtés d'autres langues internationales, comme l'anglais. Mais la principale innovation tient dans l'individuation, aux côtés de l'estonien, de quatre langues collatérales (cf. Eloy, 2004) : võro, seto, mulgi, kihnu, territorialisées dans quatre régions quasiment enchâssées les unes dans les autres, à la manière d'une poupée gigogne (à l'exception de Kihnu, langue d'une petite île distante des autres variétés méridionales). Potentiellement, les autres variétés jusqu'à maintenant considérées comme des dialectes estoniens sont susceptibles de devenir aussi des langues collatérales, selon la logique de consolidation de l'identité nationale à travers le pluralisme collatéral, ces quatre langues ayant été considérées comme des dialectes durant toute l'histoire sociolinguistique du pays, du moins de la phase II à la phase VIII, et l'Estonie nouvellement indépendante depuis 1991 
appliquant pour cette politique d'individuation collatérale les mêmes mesures qui lui ont réussi dans le passé (cf. Chalvin, 2012).

Tableau 1.7. Diglossie de la phase IX de Hennoste [Est.Eur.Glob.].

\begin{tabular}{|l|l|l|}
\hline Haute & Estonien & Référentiaire \\
\hline & Anglais, russe & Véhiculaire \\
\hline & Võro, seto, mulgi, kihnu & Collatéral \\
\hline Basse & Réseau dialectal estonien & Vernaculaire (collatéral émergent) \\
\hline
\end{tabular}

\section{Lire et interpréter les données}

Il est intéressant de comparer les pratiques langagières déclarées, pratiquement à la même époque, lors de grandes enquêtes sociolinguistiques, entre deux territoires en situation de diglossie fergusonienne : la Galice, dans la périphérie nord-occidentale de l'Espagne, et Võru, dans la périphérie méridionale de l'Estonie. Bien entendu, ces données correspondent à des usages déclarés, et non pas à des pratiques effectives observées par un enquêteur compétent. Mais elles n'en restent pas moins indicielles quant au statut, réel ou projeté, des langues en question, face à la langue dominante le référentiaire national.

Tableau 2.1. Communauté autonome de Galice (Xunta de Galicia), Espagne, 1995

\begin{tabular}{|l|l|l|l|l|l|}
\hline & $\begin{array}{l}\text { Seulement } \\
\text { espagnol }\end{array}$ & en & $\begin{array}{l}\text { Surtout en } \\
\text { espagnol }\end{array}$ & $\begin{array}{l}\text { Surtout en } \\
\text { galicien }\end{array}$ & $\begin{array}{l}\text { Seulement en } \\
\text { galicien }\end{array}$ \\
\hline A & $\begin{array}{l}\text { Classe } \\
\text { «populaire » }\end{array}$ & $6 \%$ & $11 \%$ & $27,3 \%$ & $55,7 \%$ \\
\hline B $\begin{array}{l}\text { Classe moyenne } \\
\text { basse }\end{array}$ & $6 \%$ & $14,7 \%$ & $31,6 \%$ & $47,7 \%$ \\
\hline C & Classe moyenne & $14,4 \%$ & $27 \%$ & $29,4 \%$ & $29,2 \%$ \\
\hline D $\begin{array}{l}\text { Classe moyenne } \\
\text { haute }\end{array}$ & $27,7 \%$ & $37 \%$ & $21,2 \%$ & $14,1 \%$ \\
\hline
\end{tabular}

Source : Usos lingüísticos en Galicia (U.L.G.), Real Academia Galega, Seminario de sociolingüística, p. 59.

N.B. : données recueillies en 1992, publiées en 1995.

Pour la Galice, les données du tableau 2.1 montrent clairement une situation diglossique. Les groupes de population $\mathrm{A}, \mathrm{B}, \mathrm{C}, \mathrm{D}$, sont définis de manière certes intuitive, mais notre but ne sera pas ici d'entrer dans des considérations sociologiques concernant cette région d'Espagne. 
32 Notre objectif en effet, est purement méthodologique et comparatif : ce que donnent à entendre les données du tableau 2.1, c'est une diglossie classique, où les classes «basses » $\mathrm{A}$ et $\mathrm{B}$ ne déclarent s'exprimer qu'à hauteur de $6 \%$ dans la langue nationale, ou $11 \%$ et près de $15 \%$ pour les usages mixtes à dominante espagnole (en tout, $17 \%$ de préférence pour l'espagnol pour le groupe A, $21 \%$ pour le groupe B). La classe moyenne plus aisée (groupes $C$ et $D$ ) déclare respectivement, en arrondissant les valeurs, ne s'exprimer qu'en espagnol (14,5\% et $28 \%$ : pas loin du tiers, pour ce dernier chiffre); en ajoutant les usages à dominante espagnole, de $27 \%$ et $37 \%$, on obtient respectivement $41,4 \%$ et $64,7 \%$. La tendance, que confirment les usages déclarés du galicien dans la dernière colonne de droite (classe A : 55,7 \%, classe B : 47,7 \% contre classe $\mathrm{D}: 14,1 \%)$, ressemble donc à une asymétrie des fonctions des deux langues, de type résolument diglossique. Cependant, les données se prêtent tout aussi bien à une lecture plus nuancée, qui adoucit les contours de la diglossie dans cette région d'Espagne où, si le galicien peut sans conteste se définir comme une variété «basse » sur le plan axiologique (systèmes de valeurs), il n'en garde pas moins une fonction référentiaire, telle que nous l'avons définie plus haut : sur le galicien se cristallisent des enjeux identitaires et politiques, liés à l'autonomie et à la réparation historique des sévices de la dictature franquiste et de sa forme totalitaire de centralisme, si bien que la diglossie galicienne n'est pas comparable à celle d'un créole, de l'arabe dialectal au Maghreb ou de la demotiki grecque, qui servirent de parangons pour Ferguson dans son article fondateur sur la diglossie de continuum structural (op. cit.). En effet, en décalant le point de vue (et c'est précisément ce que nous voulons faire ici), le fait que la classe $C$ (la classe moyenne) déclare à près de $30 \%$ s'exprimer « surtout en galicien », et dans les mêmes proportions "seulement en galicien", donne un taux de $60 \%$ (58,6\%, sans arrondir, comme somme de 29,4 et de 29,2). Dès lors, les $27 \%$ d'usages déclarés « surtout en espagnol » que nous venons de considérer comme des indices diglossiques, peuvent tout aussi bien se prêter à une interprétation plus optimiste : s'ajoutant aux $60 \%$ d'usages à dominante galicienne que nous venons de regrouper, on pourrait tout aussi bien dire que $87 \%$, donc près de $90 \%$, autrement dit, la quasi-totalité de la classe moyenne déclare s'exprimer peu ou prou en galicien - en tous cas, déclare le connaître et le mettre en pratique à des degrés divers, sur une échelle d'intensité variable, formatée par le questionnaire sociolinguistique. Car, à négliger le fait que l'usage «surtout en espagnol » implique au moins une certaine proportion d'usage en galicien (c'est d'ailleurs bien en cela que cet usage déclaré diffère de "seulement en espagnol »), l'interprétation des données perdrait en finesse. Dès lors, l'usage déclaré par la « classe moyenne haute ", avec $37 \%$ "surtout en espagnol ", s'ajoutant à $21,2 \%$ "surtout en galicien » et $14,1 \%$ «seulement en galicien », est fortement indiciel d'une fonction référentiaire $\mathrm{du}$ galicien, au-delà du statut vernaculaire. Or, lors de la réalisation des enquêtes, en 1992, cette fonction référentiaire était appuyée depuis au moins 1986 par un aménagement linguistique volontariste dans le cadre des autonomies et de la transition démocratique. En somme, nous venons de voir que deux diagnostics très différents, mais en même temps complémentaires, peuvent être portés sur une situation de diglossie fergusonienne, notamment lorsqu'elle connaît une transition de type collatéral : les données du tableau 2.1 peuvent aussi bien s'interpréter comme une situation de vernacularité que comme l'émergence d'une collatéralité, à partir d'une adhésion référentiaire à la langue minoritaire.

Ce détour par une situation minoritaire dans une Espagne en transition démocratique était nécessaire pour contextualiser les données recueillies dans la région Võru plus ou 
moins à la même époque, également en situation de transition démocratique, au sortir de la phase VIII, là encore dans le contexte d'un aménagement linguistique émergent (cf. la fondation et l'activité du Vorru Instituut). L'échelle de stratification sociale retenue ici par les concepteurs de l'enquête est le niveau d'éducation, également réparti sur une gamme de quatre niveaux : bas ou moyen (Bac ?), moyen $(\mathrm{Bac}+2 \text { ? })^{12}$, intermédiaire entre moyen et haut et haut niveau.

Tableau 2.2. Usage de la langue võro en Estonie méridionale, 1992. $\mathrm{VIT}^{13}$.

\begin{tabular}{|l|l|l|l|l|l|}
\hline & A & B & C & D & \\
\hline & $\begin{array}{l}\text { Niveau } \\
\text { d'éducation bas } \\
\text { ou moyen }\end{array}$ & $\begin{array}{l}\text { Niveau } \\
\text { moyen }\end{array}$ & $\begin{array}{l}\text { Niveau intermédiaire } \\
\text { entre moyen et haut }\end{array}$ & $\begin{array}{l}\text { Haut niveau } \\
\text { d'éducation }\end{array}$ & Total \\
\hline Régulièrement & $63,9 \%(94)$ & $\begin{array}{l}32,2 \% \\
(48)\end{array}$ & $28,3 \%(58)$ & $15,9 \%(14)$ & $\begin{array}{l}36,3 \% \\
(214)\end{array}$ \\
\hline Occasionnellement & $27,2 \%(40)$ & $49 \%(73)$ & $48,8 \%(100)$ & $53,4 \%(47)$ & $\begin{array}{l}44,1 \% \\
(260)\end{array}$ \\
\hline Jamais & $7,5 \%(11)$ & $\begin{array}{l}14,8 \% \\
(22)\end{array}$ & $20 \%(41)$ & $23,9 \%(21)$ & $\begin{array}{l}16,1 \% \\
(95)\end{array}$ \\
\hline Autre & $1,4 \%(1,4)$ & $4 \%(6)$ & $2,9 \%(6)$ & $6,8 \%(6,8)$ & $\begin{array}{l}3,4 \% \\
(20)\end{array}$ \\
\hline Total & $25 \%(147)$ & $\begin{array}{l}25,3 \% \\
(149)\end{array}$ & $34,8 \%(205)$ & $14,9 \%(88)$ & $\begin{array}{l}(589) \\
(00)\end{array}$ \\
\hline
\end{tabular}

(Entre parenthèses sont indiqués les échantillons de population interrogés durant l'enquête.)

34 La lecture des chiffres se prête à un éventail d'interprétations : une telle matrice de données ne se laisse pas réduire à une analyse univoque. Une comparaison hâtive entre la colonne $\mathrm{A}$ et la colonne $\mathrm{D}$ pousserait à diagnostiquer une situation de diglossie : intensité des usages $(63,9 \%$, autrement dit près de $65 \%$, d'usages déclarés comme réguliers et $27,2 \%$, autrement dit près de $30 \%$, pour un usage occasionnel, soit près de $95 \%$ d'usage variable pour le groupe le moins instruit, contre près de $16 \%$ d'usage régulier pour les plus instruits). Certes, la dernière colonne à droite, celle de la moyenne des taux d'un groupe à l'autre, laisse voir que la langue est encore dotée d'une indéniable vitalité $(36,3+44,1 \%$ d'usage "régulier » et "occasionnel », soit $80,4 \%$ d'usage du võro, ce qui est une performance remarquable pour un vernaculaire ayant traversé l'exode de la période de l'annexion brutale de l'Estonie par l'Union soviétique, la collectivisation, les déportations ${ }^{14}$, l'exode rural, dans une région périphérique d'Estonie, représentant $14 \%$ du territoire du pays, et $8 \%$ de la population totale.

Mais le plus paradoxal de cette matrice de données, c'est la gamme intermédiaire des groupes B et C (niveaux d'éducation moyen et intermédiaire entre moyen et haut), qui déclare pour près du tiers $(32,2 \%$ et $28,3 \%)$ faire un usage quotidien de la langue võro, et pour moitié (49 et $48,8 \%$ ) l'utiliser occasionnellement. Les groupes B, C et D sont en relation complémentaire, relativement asymétrique, avec le groupe $\mathrm{A}$ : là où ce dernier 
déclare ne parler occasionnellement le võro que pour $27,2 \%$ des personnes interrogées, les trois autres groupes plus instruits déclarent l'utiliser occasionnellement à près de $50 \%$, ce qui est un compromis remarquable en faveur de la langue locale. Selon notre grille critique, tout se passe comme si l'usage devenait de moins en moins corporatiste (cette fois dans le sens de la ghettoïsation vernaculaire, caractéristique de la diglossie, mais en faisant référence aux milieux agricoles de cette région rurale, sur le plan sociologique), et de plus en plus référentiaire, exprimant un attachement au contexte local. Le võro tend à passer, par conséquent, de sociolecte rural stigmatisé, à une langue régionale de facto - sinon de statut, du moins à teneur référentiaire sur le plan psychosocial-, sans négliger des avancées effectives vers sa reconnaissance régionale de jure. Une telle asymétrie peut, en situation de contact entre langues proches (estonien standard versus langue collatérale) s'interpréter en termes de différence de compétence linguistique: les locuteurs ayant vécu l'expérience de la vernacularité s'avérant meilleurs locuteurs que ceux qui sortent d'une phase d'acculturation induite par le contexte diglossique qui a prévalu jusqu'alors, et dont l'amélioration des conditions sociales (notamment le niveau d'éducation plus élevé) a été la motivation pour moins investir dans une compétence bilingue langue dominante/langue dominée. $\mathrm{Au}$ moment où le statut de la langue minorée devient davantage référentiaire et gratifiant (amélioration de statut), les groupes et les classes d'âge qui en ont été tenus à l'écart ou qui s'en sont écartés amorcent un retour à la langue, qui se fait par étapes et par niveaux d'immersion étagés, au gré des interactions en face à face, selon les interlocuteurs, mais aussi selon les thèmes de conversation. C'est ce qu'on peut observer en Galice, à la lumière des données exposées plus haut, et c'est également ce qui semble se passer, au début des années 1990, en région Võru. Heureusement, les données dont nous disposons nous permettent d'observer ce phénomène de plus près, en termes de gamme de compétences linguistiques, au sein du répertoire pluraliste émergent (estonien standard/continuum diaphasique ${ }^{15}$ võro). Bien que le détail de la variation diaphasique ne soit pas ici accessible, il importe de garder à l'esprit que cette modalité doit faciliter ou orienter les choix de type 1 (Je parle couramment, partout et de tout en langue vorro), 2 (Je parle couramment, à la maison et au sujet des choses de la vie quotidienne, en langue vorro), etc.

Tableau 2.3. Usage de la langue võro en Estonie méridionale, fréquence d'usage et compétence linguistique déclarées, $1992^{16}$.

\begin{tabular}{|c|c|c|c|c|c|c|c|}
\hline & & 1 & 2 & 3 & 4 & 5 & \\
\hline & & $\begin{array}{l}\text { Je parle } \\
\text { couramment, } \\
\text { partout, et de } \\
\text { tout en } \\
\text { langue võro }\end{array}$ & $\begin{array}{l}\text { Je parle } \\
\text { couramment, } \\
\text { à la maison et } \\
\text { au sujet des } \\
\text { choses de la } \\
\text { vie } \\
\text { quotidienne, } \\
\text { en langue võro }\end{array}$ & $\begin{array}{l}\text { Je parle } \\
\text { un peu } \\
\text { le võro, } \\
\text { quand } \\
\text { je suis } \\
\text { avec des } \\
\text { gens qui } \\
\text { le } \\
\text { parlent }\end{array}$ & 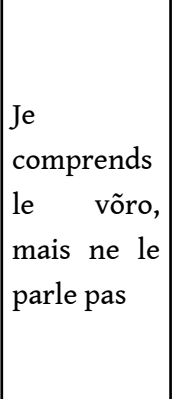 & $\begin{array}{l}\text { Je ne parle } \\
\text { ni ne } \\
\text { comprends } \\
\text { le võro }\end{array}$ & Total \\
\hline 盬 & Régulièrement & $83,6 \%$ (179) & $15 \%(32)$ & $0,9 \%(2)$ & $0,5 \%(1)$ & 0 & $\begin{array}{l}36,4 \% \\
(214)\end{array}$ \\
\hline
\end{tabular}




\begin{tabular}{|c|c|c|c|c|c|c|c|}
\hline 媐 & Occasionnellement & $27,7 \%(72)$ & $25,8 \%(67)$ & $\begin{array}{l}38,8 \% \\
(101)\end{array}$ & $7,3 \%(19)$ & $0,4 \%(1)$ & $\begin{array}{l}44,2 \% \\
(260)\end{array}$ \\
\hline 閶 & Jamais & 0 & $1,1 \%(1)$ & $6,3 \%(6)$ & $81,1 \%(77)$ & $11,6 \%(11)$ & $\begin{array}{l}16,2 \% \\
(95)\end{array}$ \\
\hline 䜿 & Autre & 0 & $5,3 \%(1)$ & $\begin{array}{l}26,3 \% \\
(5)\end{array}$ & $68,4 \%(13)$ & 0 & \begin{tabular}{|l}
$3,2 \%$ \\
$(19)$
\end{tabular} \\
\hline 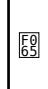 & Total & $42,7 \%(251)$ & $17,2 \%(101)$ & $\begin{array}{l}19,4 \% \\
(114)\end{array}$ & $\begin{array}{l}18,7 \% \\
(110)\end{array}$ & $2 \%(12)$ & $\begin{array}{l}100 \% \\
(508)\end{array}$ \\
\hline
\end{tabular}

(Entre parenthèses sont indiqués les échantillons de population interrogés durant l'enquête.)

Si l'on définit les colonnes 1 et 2 du tableau 2.3 comme porteuses d'indices d'usage vernaculaire du võro, ces données confirment la tendance à un usage vernaculaire du võro (cf. cellules $\alpha-1: 83,6 \%, \beta-1: 27,7 \%, \alpha-2: 15 \%$, et $\beta-2: 25,8 \%$ ), avec un total de 42,7 $+17,2 \%=$ env. $60 \%$ d'usages vernaculaires du võro (cellules $\varepsilon-1$ et $\varepsilon-2$ ). Les colonnes 3 et 4 constituent un champ de disponibilité et de volatilité des usages - mais aussi une certaine marge de tolérance à l'emploi actif ou passif de la langue - : les cellules $\beta-3$ ( $38,8 \%$ d'usage conditionné aux réseaux de compétence active en langue võro, soit près de $40 \%$ des usages occasionnels. Les données de la colonne 4 s'expliquent par une étiquette (dans le sens d'ethos) de la répartition des usages, caractéristique des situations de diglossie à référentiaire rural fort : en $\gamma-4$ 81,1\% des personnes interrogées qui se reconnaissent une compétence passive déclarent ne jamais essayer de le parler. Mais comment interpréter la cellule $\delta-4$ ( $68,4 \%$ préférant pointer la réponse «autre stratégie de communication $»$ ? ${ }^{17}$

Le tableau 2.4 va nous permettre de mieux cerner cette dynamique relationnelle, cette fois en maintenant la stratification sociale qui nous a servi de point de départ. Les cellules 1-A et 2-A ainsi que 1-B et 2-B confirment le statut vernaculaire de la langue locale, et l'attitude plutôt favorable au võro de la part des classes intermédiaires et élevées (C et D), puisque le tiers $(33,8 \%)$ ou près du tiers $(28,4 \%)$ de ces deux groupes déclare le parler "couramment et en tous lieux». Mais les données les plus intéressantes, au-delà des proportions attendues, résident dans des asymétries qui dénotent des choix riches en indices sur la trame des compétences : en 4-A, 10,9\% des locuteurs d'extraction modeste se déclarent «bilingues passifs» (comprend le vorro, mais ne le parle pas), et cette proportion va en augmentant, de la catégorie B (niveau moyen) à la catégorie $\mathrm{D}$ (haut niveau éducatif), jusqu'à atteindre un bon quart de la population concernée $(26,1 \%)$. Or, la nature des compétences passives est très probablement différente d'un groupe à l'autre: dans le groupe A, il s'agit sans doute de locuteurs acculturés ou de la génération que leurs parents d'origine modeste ont voulu " préserver » de l'immersion vernaculaire - ou qui ont rejeté le võro comme " ringard " ou inutile -, tandis que pour les groupes B, C et D (respectivement $18,1 \%, 21,6 \%$ et $26,1 \%$ ), il est probable que plus le niveau d'instruction est élevé, plus les personnes sont activement disposées à apprendre le võro, du moins suffisamment pour le comprendre, notamment par adhésion à sa fonction référentiaire locale. En d'autres termes, les uns sont bilingues passifs parce qu'ils souhaitent oublier le võro, tandis que les autres le sont tout en restant, au contraire, disposés à l'apprendre, ou parce qu'ils 
sont en train de l'apprendre. Les classes les plus élevées, pourtant moins aptes apparemment à parler activement le võro, semblent être la locomotive qui tire cette langue vers le haut : de la fonction vernaculaire à la fonction véhiculaire.

Tableau 2.4. Usage de la langue võro en Estonie méridionale, niveau d'éducation et compétence linguistique déclarée, $1992^{18}$.

\begin{tabular}{|l|l|l|l|l|l|}
\hline & A & B & C & D & 席 \\
\hline & $\begin{array}{l}\text { Niveau } \\
\text { d'éducation bas ou } \\
\text { moyen }\end{array}$ & $\begin{array}{l}\text { Niveau } \\
\text { moyen }\end{array}$ & $\begin{array}{l}\text { Niveau intermédiaire } \\
\text { entre moyen et haut }\end{array}$ & $\begin{array}{l}\text { Haut niveau } \\
\text { d'éducation }\end{array}$ & Total \\
\hline $\begin{array}{l}\text { 1. Couramment et } \\
\text { en tous lieux }\end{array}$ & $63,9 \%$ & $42,3 \%$ & $33,8 \%$ & $28,4 \%$ & $42,7 \%$ \\
\hline $\begin{array}{l}\text { 2. Couramment à la } \\
\text { maison }\end{array}$ & $17,7 \%$ & $16,1 \%$ & $18,6 \%$ & $14,8 \%$ & $17,2 \%$ \\
\hline 3. Un peu, selon \\
l'interlocuteur
\end{tabular}

(L'échantillon est analogue à celui du tableau 2.2 supra, correspondant au tableau 1.7 du VIT.)

\section{Conclusion}

Nous terminerons par conséquent cette brève étude sur une conclusion simple: la dimension quantitative n'est rien sans une théorie interprétative des données, sans catégorisations articulées en un système ou un modèle. Lire des données statistiques sans une connaissance du terrain et sans vision dynamique entre phases successives, du point de vue de l'histoire sociolinguistique d'une situation de contact de langues, sans une lecture herméneutique, permet certes de saisir de grandes tendances, mais ne permet pas de rendre compte des enjeux sociétaux et glottopolitiques. Nous avions présenté, lors d'un colloque en Estonie, cette forme d'analyse interprétative appliquée au mazatec (Léonard 2012). Nous avions ensuite esquissé, dans l'Hexagone, une approche herméneutique de la question des langues collatérales du sud de l'Estonie (Léonard, 2013). Nous avons tenté ici d'opérer une synthèse de ces deux approches, sur des terrains très divers, afin de contribuer, dans le cadre très précis de ce volume, qui contient de nombreuses contributions fondées sur la lecture de données quantitatives, à une réflexion aussi bien méthodologique qu'épistémologique critique sur ce que nous 
disent les locuteurs sur la dynamique sociolinguistique de leur relation aux langues, lorsqu'ils répondent à un questionnaire déclaratif.

\section{BIBLIOGRAPHIE}

BertricAu A. et Chalvin Antoine (sous la direction de), 2001, L'Estonie. Identité et indépendance, Paris, L'Harmattan.

CANUT Cécile, 2008, Une langue sans qualité, Limoges, Lambert Lucas.

CHALVIN Antoine, 2010, Johannes Aavik et la rénovation de la langue estonienne, coll. Bibliothèque finno-ougrienne, Paris : ADEFO / L'Harmattan.

CHALvin Antoine, 2012, « La construction de l'identité des Setos (1920-1940) », Études finnoougriennes, 43 : p. 119-144.

FORNEL Michel de, OGIEN Albert, QUÉRÉ Louis (sous la direction de), 2001, L'ethnométhodologie : une sociologie radicale [Colloque de Cerisy], Paris, La Découverte.

ELOY Jean-Michel (sous la direction de), 2004, Des langues collatérales. Problèmes linguistiques, sociolinguistiques et glottopolitiques de la proximité linguistique, actes du colloque international d'Amiens, 21-24 novembre 2001, 2 volumes, Paris, L'Harmattan.

FERGUSON Charles, 1959, “Diglossia”, Word, 15.

HenNoste Tiit, 1997, „Eesti sotsioperioodid. Üldpilt“, Pühendusteos Huno Rätsepale, Tartu, Publications de la chaire d'estonien de l'université de Tartu, nº 7, p. 45-66.

KULMAR Tarmo, 2001, «Les habitants de l'Estonie préhistorique », in Bertricau et Chalvin 2001, p. 37-50.

LAAR Mart, 2002, «L'Estonie et le communisme », in CouRToIs Stéphane, 2002, Du passé faisons table rase ! Histoire et mémoire du communisme en Europe, Paris, Robert Robert Laffont, p. 395-546 (traduit par Jean-Pierre Minaudier).

LÉONARD, Jean Léo, 2004, « Langues fenniques collatérales en ex-URSS : vepse, carélien, olonetsien (Carélie russe) et võro-seto (Estonie et région de Pskov)», in Eloy 2004, p. 575-592.

LÉONARD Jean Léo, 2012, “Mazatec (Popolocan, Eastern Otomanguean) as a Multiplex Sociolinguistic 'Open Small World”', colloque international Languages of Smaller Populations: Risks and Possibilities, Tallinn, 16-17 mars 2012.

LÉONARD Jean Léo, 2013, « Émergence de langues proches fenniques et reconfiguration des répertoires langagiers en Estonie ", à paraître dans les actes du colloque Gestion des Minorités linguistiques en Europe, Université de Montpellier 3.

Patriarca M., Castelló X., URiarte J.R., Eguíluz V.M. and San Miguel M., 2012, “Modelling TwoLanguage Competition Dynamics", Advances in Complex Systems Vol. 15, no 3-4.

PATRIARCA M., HeINSALU E., 2008, "Influence of geography on language competition”, Physica A 388 (2009), pp. 174-186. 
TARVEL Enn (éd.) et KLEIS Richard (trad.), 1982, Heinrici Chronicon Livoniae / Henriku Liivimaa

kroonika, Tallinn, Eesti Raamat.

Données

[ULG] Usos lingüísticos en Galicia, Real Academia Galega, Seminario de sociolingüística, p. 59.

[VIT] Võro Instituut, 2000. A kiilt rahvas kynõlõs... Võrokeste keelest, kommetest, identiteedist, Võro Instituudi Toimõtiseq, $\mathrm{n}^{\circ} 8$.

\section{NOTES}

1. Les idées présentées ici sont issues d'une communication au colloque « Gestion des minorités linguistiques en Europe ", tenu en décembre 2012 à Montpellier (Léonard 2013), et surtout du dernier cours de Master 2 «Construction nationale et aménagement linguistique » (2012-13), qui se tenait depuis 2003 à l'ILPGA, Paris 3. Nous avons bien écrit dernier cours, puisqu'en raison des contraintes budgétaires appliquées aux universités françaises avec de plus en plus de rigueur, cet enseignement sera supprimé à partir de l'année 2013-2014. Cet article est donc dédié aux étudiants qui l'ont suivi et qui ont contribué à alimenter sa méthodologie depuis près de dix ans, notamment par la rédaction de dossiers de recherche sur le microaménagement linguistique. Par ailleurs, la dette intellectuelle que nous avons à l'égard de Els Heinsalu et de Marco Patriarca, physiciens travaillant sur la modélisation des processus de bilinguisme et de contact de langues, est immense (cf. Patriarca et coll. 2012, Patriarca et Heinsalu 2008). Toute erreur éventuelle dans l'interprétation des modèles aussi bien que des données incombe à nous seul. Nous remercions également Eva Toulouze (Inalco, E.F.O.) pour sa patience et son empathie en tant que co-éditrice de ce volume.

2. Cf. Kurman 1968, pour une synthèse très documentée sur l'histoire des textes en estonien nord et sud, qui se prêterait à une projection chronologique plus fine, sans remettre en cause pour autant les grandes lignes tracées par Hennoste. L'essai d'Antoine Chalvin (2010) reste la référence incontournable en matière d'histoire de l'élaboration linguistique de l'estonien, notamment pour les phases IV à VII de Hennoste. Par ailleurs, l'ouvrage collectif coordonné par A. Bertricau et Antoine Chalvin (2001) est riche en articles éclairant des points précis de la chronologie reprise ici à Hennoste.

3. On distingue la diglossie de Ferguson, qui concerne le bilinguisme dialectal en situation de continuum structural (créole à base lexicale française versus français standard, arabe dialectal versus arabe littéraire, demotiki versus katharevusa, etc.), de la diglossie de Fishman, qui s'applique aux langues qui ne sont pas en relation de continuum structural (espagnol versus anglais, estonien versus bas-allemand ou haut-allemand, estonien versus russe, etc.).

4. Nous reprenons ici, en les résumant et en les traduisant en français, les critères présentés par Tiit Hennoste dans son article de 1997, en appliquant des notions de sociolinguistique générale, dans la mesure où le détail de sa périodisation décrit avant tout l'histoire sociale de l'estonien. Nous avons également utilisé la synthèse publiée sur Internet par Helen Allik.

5. Cf. Kulmar (2001).

6. La variation diastratique est le champ des sociolectes, ou variétés de groupes sociaux distingués par des critères de classe ou de caste, de même que la variation diatopique est le champ de la diversité géolinguistique, et que la variation diaphasique est le champ de la variation entre registres stylistiques, selon le contexte plus ou moins formel de la communication. Ces notions sont désormais triviales en sociolinguistique.

7. Versuch ueber den werroehstnischen dialekt / Uurimus võru murdest, Tartu, Tartu ülikooli õppetooli toimetised, 20, trad. estonienne de Inge Annom. 
8. La dilalie diffère de la diglossie par son caractère relativement neutre - moins inégalitaire, ou pas nécessairement fondé sur une hiérarchisation stricte des normes ou variétés des répertoires - de coexistence de registres au sein d'un répertoire: cf. Berruto, p. 242-250. Les registres littéraires (confessionnel, médiatique) et les variétés d'estonien dialectal ainsi que les « interlectes » estoniens plus ou moins dialectaux ou standardisés que parlaient les germanobaltes, notamment dans les phases III surtout, et peut-être aussi IV, entretenaient probablement une relation de dilalie davantage que de diglossie à proprement parler.

9. Ces remarques proviennent principalement de la synthèse d'Helen Allik.

10. Cependant, l'exercice serait possible, en fondant l'analyse sur la grande richesse d'indices et de données rapportées par un important document historique datant du XIII ${ }^{\mathrm{e}}$ siècle : la chronique d'Henri de Livonie (cf. l'édition par Enn Tarvel et Richard Kleis, 1982). Nous ne nous y risquerons pas dans le cadre de cet exposé succinct, qui se veut purement méthodologique.

11. Sur la question de savoir comment qualifier les langues, notre réflexion se situera dans une zone neutre par rapport à l'essai de Cécile Canut Une langue sans qualité (Canut, 2008), qui se prononce en faveur d'une neutralisation des projections essentialistes de tous types sur les langues. Certes, les dérives de l'essentialisme sont néfastes en sociolinguistique comme en anthropologie: il n'y a ni immanence d'un quelconque esprit des langues, ni qualités intrinsèques, ni qualités données en soi. Mais que le social et le politique ne cessent de reconfigurer les rapports de force entre les langues en jouant sur leur ontologie (ce qui fait qu'un objet ou qu'un être existe) et leur axiologie (le système de valeurs qui leur est attaché) est inévitable et constitue en soi un objet d'observation critique des pratiques. Toutes les qualités projetées sur les langues ne sont pas néfastes en soi : elles peuvent le devenir sous la pression d'intérêts sectaires - et, en tant que telles, mériter une franche condamnation du linguiste pour des raisons éthiques. Mais priver l'analyse sociolinguistique de l'étude de la gamme des qualités successives, données ou construites, revient à une clôture de la pensée. Une distanciation ethnométhodologique s'impose (cf. de Fornel et coll. 2004), fertile en observations et en perspectives pour une théorie générale en sociologie du langage : une théorie qui ne s'arrête pas au seuil de la déqualification. Au contraire, requalifier les langues dans une optique de glissements de valeurs et de fonctions est un exercice salutaire pour la sociolinguistique moderne, qui se trouve sans cesse confrontée à des qualités polyvalentes, polysémiques ou à géométrie variable.

12. Sauf erreur de notre part, les niveaux d'éducation ne sont pas spécifiés avec précision dans notre source.

13. Source: Võro Instituut, 2000. Võro Instituudi Toimõtiseq, $\mathrm{n}^{\circ} 8, \mathrm{p} .146$, tableau 1.7. (Données recueillies au début des années 1990 , publiées en 2000.)

14. Quelques chiffres sur cette question, issus de la synthèse de Mart Laar (2002: p. 430-490) : cet auteur rappelle que la population de l'Estonie, qui s'élevait à 1000360 Estoniens en 1939, n'était plus que de 907222 fin 1941, puis de 806000 fin 1944. En cinquante ans, de 1939 à 1989, la population estonienne a baissé de 117000 personnes, alors que le nombre aurait dû augmenter de 313000 personnes. Il faut transposer ces pertes démographiques en proportion avec des pays plus peuplés, comme le rappelle M. Laar (op. cit., p. 468) pour saisir l'ampleur du désastre démographique et humain. Ces données générales devraient être complétées avec des données spécifiques au sud de l'Estonie, dont nous ne disposions pas lors de la rédaction de cette contribution.

15. La variation diaphasique correspond à la variation stylistique : prédominance d'éléments basilectaux (plus proches de la variété vernaculaire) et acrolectaux (plus proches de la variété standard, ici principalement l'estonien).

16. Source : idem, p. 149, tableau 1.11 de l'ouvrage.

17. L'une de ces stratégies pourrait bien être le silence, afin de laisser la personne parlant võro utiliser sa variété le plus possible sans interférer avec l'usage de l'estonien standard. Voici 
typiquement un exemple de case défective dans un questionnaire sociolinguistique, qui passe à côté d'un complexe de relations sociales d'un grand intérêt.

18. Source : idem, p. 151, tableau 1.13 de l'ouvrage.

\section{RÉSUMÉS}

Les situations sociolinguistiques évoluent considérablement au cours du temps. Loin de se cantonner à un conflit figé ou à une situation de subordination prédéterminée, la diglossie, par exemple, y compris sous sa forme continuiste (diglossie de Ferguson), peut changer de forme et de contenu en quelques générations, au gré des conditions glottopolitiques et sociales, comme le montre le cas des quatre langues collatérales d'Estonie méridionale (võro, seto, mulgi, kihnu). Par ailleurs, la lecture qui est faite des données quantitatives s'avère souvent sommaire : l'analyste se contente de regarder de grandes proportions pour arriver à des conclusions hautement prédictibles, sans que l'on sache si une logique de falsification popperienne guide sa démarche, ou simplement la doxa. Enfin, les concepts qu'utilisent les sociolinguistes sont souvent a prioristiques au point de confiner à la doxa. Une manière de résorber ces apories et ces contradictions consiste à revisiter de manière critique les notions de diglossie, de vernacularité et de véhicularité, liées au statut ou au prestige des langues ou variétés constitutives des répertoires bilingues ou multilingues ds locuteurs, à travers une analyse des conditions historiques d'émergence de ces registres linguistiques en contact dans un champ social dynamique, tout en essayant de diversifier les concepts et de les articuler en un modèle qui puisse profiter aussi bien à la modélisation qu'à l'analyse qualitative des données statistiques sur les pratiques langagières.

Socio-linguistic situations vary throughout time. The form and content of diglossia, for instance, while not being limited to rigid conflict or to a predestined subordination, (even in its continuist form, alias Ferguson's diglossia) may change in some generations, depending on political and social conditions, as is clear in the case of the four southern Estonian collateral languages (Võro, Seto, Mulgi, Kihnu). Moreover, often this change comes from an interpretation of quantitative data: the analyst just observes the proportions at a larger scale in order to come to highly predictable conclusions, while nobody knows whether he or she is guided by a Popper-like falsification approach or only by doxa. Finally, the concepts used by the sociolinguists often rely on a priori knowledge, so that these concepts frequently become similar to doxa. How to solve these difficulties and contradictions? One way would be to revisit critically different notions diglossia, vernacularism, vehicularity, issues connected with the status or prestige of the speakers' bilingual or multilingual language or repertoires - by analysing the historical conditions of the emergence of these language registers on a dynamic social field. Meanwhile, we attempt to diversify the concepts and to articulate them in a model that would be useful both to modelisation and to qualitative analysis of language practise's statistical data.

Sotsiolingvistilised situatsioonid varieeruvad aja jooksul märgatavalt. Näiteks diglossia vorm ja sisu - mis ei piirdu jäiga konflikti ega ettemääratud allutavussuhtega, seal hulgas kontinuistlike vormide puhul (Fergusoni diglossia) - võivad muutuda mõne põlvkonna jooksul, sõltudes keelepoliitilistest ja sotsiaalsetest tingimustest, nagu näitab nelja Lõuna-Eesti kollateraalse keele (võro, seto, mulgi, kihnu) näide. Lisaks sellele loetakse kvantitatiivseid andmeid sageli väga 
ligikaudselt: uurija vaatleb vaid üldproportsioone ja jõuab nende kaudu suuresti ette ennustatavate järeldusteni, nii et ei ole mõistetav, kas ta juhindub popperlikust falsifikatsioonist või ainult üldtõdedest. Pealegi põhinevad sotsiolingvistide mõisted sageli aprioorsetel alustel, mis sarnanevad üldtõdedega. Üks võimalikke viise nimetatud aprioorsusi ja vastukäivusi lahendada seisneb lähenemises, kus mõisted diglossia, vernakulaarsus, ühiskeel vaadatakse kriitilise pilguga üle. Need on seotud keelekõnelejate kakskeelse või mitmekeelse staatuse või prestiižiga. Neid tuleb analüüsida lähtudes vaatlusaluste keelte registrite tekke ajaloolistest tingimustest dünaamilise ühiskondliku tausta raames. Samal ajal tuleb püüda mõisteid mitmekesistada ja liigendada need mudeliks, millest võiks olla abi keelepruugi kohta käivate andmete modelleerimisel ja kvalitatiivsel analüüsil.

\section{INDEX}

Keywords : Estonia, Estonian, Dialects, Collateral Languages, sociolinguistics, language practice, qualitative sociology, modelisation

nomsmotscles peuples baltes, peuples scandinaves, peuples fenniques, peuples slaves, Danois, Suédois

disciplines allemand, anglais, arabe dialectal, bas-allemand, créole, demotiki grac, espagnol, estonien, français, galicien, haut-allemand, italien, kihnu, langues fenniques, langues ouraliennes, latin, mazatec, mulgi, picard, russe, setu, slavon, suédois

Mots-clés : dialectes, langues collatérales, sociolinguistique, méthodologie, pratiques langagières, sociologie qualitative, sociologie quantitative, modélisation

Thèmes : sociolinguistique

Index géographique : Galice, France, Kihnu, Mulgimaa, Saaremaa, Tallinn, Tartu, Võru, Sibérie 